\title{
Dispersion relation of lipid membrane shape fluctuations by neutron spin-echo spectrometry
}

\author{
Maikel C. Rheinstädter ${ }^{1}$ ه Wolfgang Häußler ${ }^{2}$, and Tim Salditt ${ }^{3}$ \\ ${ }^{1}$ Institut Laue-Langevin, 6 rue Jules Horowitz, BP 156, 38042 Grenoble Cedex 9, France \\ ${ }^{2}$ FRM-II, Technische Universität München, Lichtenbergstraße 1, 85747 Garching, Germany \\ ${ }^{3}$ Institut für Röntgenphysik, Friedrich-Hund Platz 1, 3707 Göttingen, Germany
}

(Dated: September 8, 2018)

\begin{abstract}
We have studied the mesoscopic shape fluctuations in aligned multilamellar stacks of DMPC bilayers using the neutron spin-echo technique. The corresponding in plane dispersion relation $\tau^{-1}\left(\mathrm{q}_{\|}\right)$at different temperatures in the gel (ripple, $\left.\mathrm{P}_{\beta^{\prime}}\right)$ and the fluid $\left(\mathrm{L}_{\alpha}\right)$ phase of this model system has been determined. Two relaxation processes, one at about $10 \mathrm{~ns}$ and a second, slower process at about 100ns can be quantified. The dispersion relation in the fluid phase is fitted to a smectic hydrodynamic theory, with a correction for finite $\mathrm{q}_{z}$ resolution. We extract values for, the bilayer bending rigidity $\kappa$, the compressional modulus of the stacks $B$, and the effective sliding viscosity $\eta_{3}$. The softening of a mode which can be associated with the formation of the ripple structure is observed close to the main phase transition.
\end{abstract}

PACS numbers: 87.16.Dg, 83.85.Hf, 62.20.Dc

Thermally excited shape fluctuations of membranes are readily observed in the light microscope, e.g., the flickering of lipid vesicles or biological membranes. On the nanometer scale, undulations of the lipid bilayer are experimentally much more difficult to probe. Few spectroscopic techniques offer the spatial and temporal resolution needed for the validation of the theoretic work on collective bilayer motion, see e.g., 1, 2, 3, 4, 5]. Up to now, thermal fluctuations of lipid membranes, in particular in multilamellar stacks, have been mainly investigated by thermal diffuse scattering, i.e., x-ray lineshape analysis [6, 7]. Elastic scattering has led to a detailed understanding of the static properties of thermal fluctuations in lipid membranes and the elasticity properties governing these fluctuations. According to linear smectic elasticity theory [8, 9] thermal fluctuations in the fluid phase of the membrane are governed by the free energy functional (Hamiltonian)

$$
H=\int_{A} d^{2} r \sum_{n=1}^{N-1}\left(\frac{1}{2} \frac{B}{d}\left(u_{n+1}-u_{n}\right)^{2}+\frac{1}{2} \kappa\left(\nabla_{\|}^{2} u_{n}\right)^{2}\right),
$$

where $\kappa$ denotes the bilayer bending rigidity, $A$ the area in the $x y$-plane, $N$ the number of bilayers, and $u_{n}$ the deviation from the average position $n d$ of the $n$-th bilayer, $d$ is the lamellar spacing. $B$ and $K=\kappa / d$ are elastic coefficients, governing the compressional and bending modes of the smectic phase, respectively. A fundamental length scale in these systems is given by the smectic penetration length $\Lambda=\sqrt{K / B}$. Aligned lipid bilayers allow a separate determination of both parameters $K$ and $B[10,11$. However, a full understanding of the collective dynamics should include experimental determination of relaxation rates, the relevant transport coefficients (viscosities), and in particular the characteristic dispersion relation of the relevant modes.
In this letter we present an experimental dispersion relation for aligned multilamellar lipid membranes $\tau^{-1}\left(q_{\|}\right)$ as a function of lateral momentum transfer $q_{\|}$(in the plane of the bilayers), measured by neutron spin-echo spectrometry (NSE). From analysis of the dispersion relation, the effective sliding viscosity, $\eta_{3}$, as well as the static properties $\kappa$ and $\Lambda$ are obtained. A softening of a mode which can be associated with the formation of the ripple structure is observed close to the main phase transition. In the $q_{\| \mid}$range probed, the bilayer displacement $u_{n}$ can be assumed to be a continuous variable. It is therefore not sensitive to the discrete molecular structure, in contrast to the dynamics on the molecular length scale (acyl chain distance) which can be probed by inelastic neutron scattering using three-axes spectrometry [12]. We have selected NSE for this study since the undulation modes at high $q_{\|}$are too fast to be accessed by x-ray photon correlation spectroscopy (XPCS) and the lateral length scales are to small to be resolved by dynamic light scattering (DLS) 13]. XPCS has successfully been used for investigations of free-standing thermotropic liquid crystals, which exhibit much slower acoustic modes, corresponding to center of mass movement of the entire film 14. NSE has been used before to study phospholipid membranes, 15, 16]. However, a dispersion relation over a wide range in $q_{\|}$in the fluid multilamellar state has not been achieved so far, in part due to flux and technical limitations in the past.

NSE directly measures the intermediate scattering function $\mathrm{S}(\mathbf{q}, \mathrm{t})$, which is related to the density distribution $\rho(\mathbf{r}, t)$ in the sample by

$$
S(\mathbf{q}, t)=\int d^{3} \mathbf{R} e^{-i \mathbf{q} \mathbf{R}} \int d^{3} \mathbf{r}\langle\rho(\mathbf{r}, 0) \rho(\mathbf{r}+\mathbf{R}, t)\rangle .
$$

The spin-echo experiments were carried out at the IN11 and IN15 spectrometers, situated at the cold source of the high flux reactor of the Institut Laue-Langevin (ILL) 

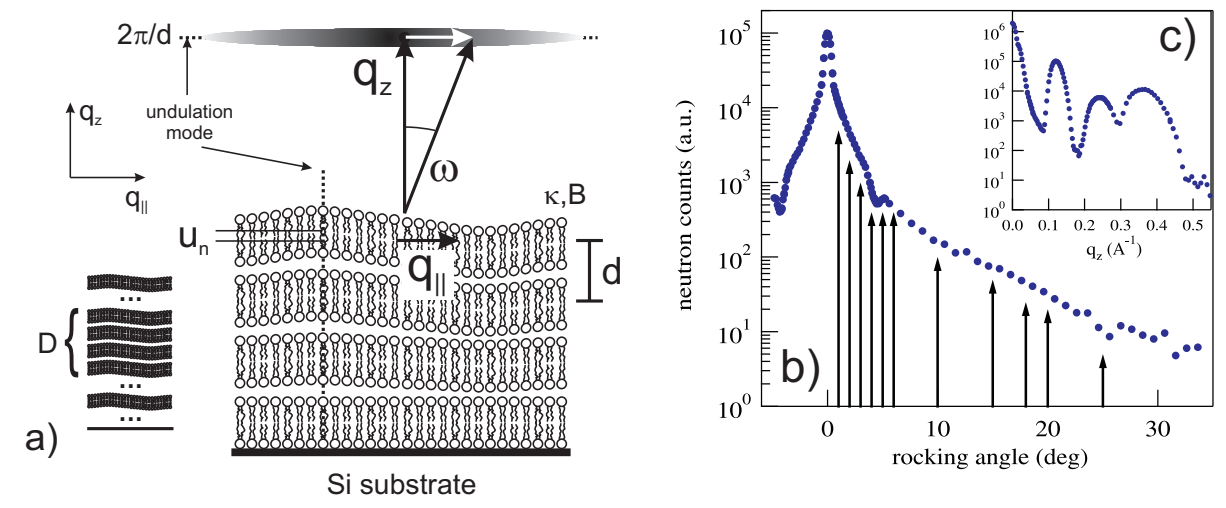

FIG. 1: (Color online). (a) Sketch of the scattering geometry. (b) Rocking scan at $\mathrm{T}=30^{\circ} \mathrm{C}$ through the first Bragg peaks and along the diffuse Bragg sheet in log-log scaling. The arrows exemplary mark positions of inelastic scans in the fluid phase. (c) Inset shows a reflectivity curve as measured on IN11 with relaxed momentum resolution $(\Delta \lambda / \lambda \simeq 15 \%)$.
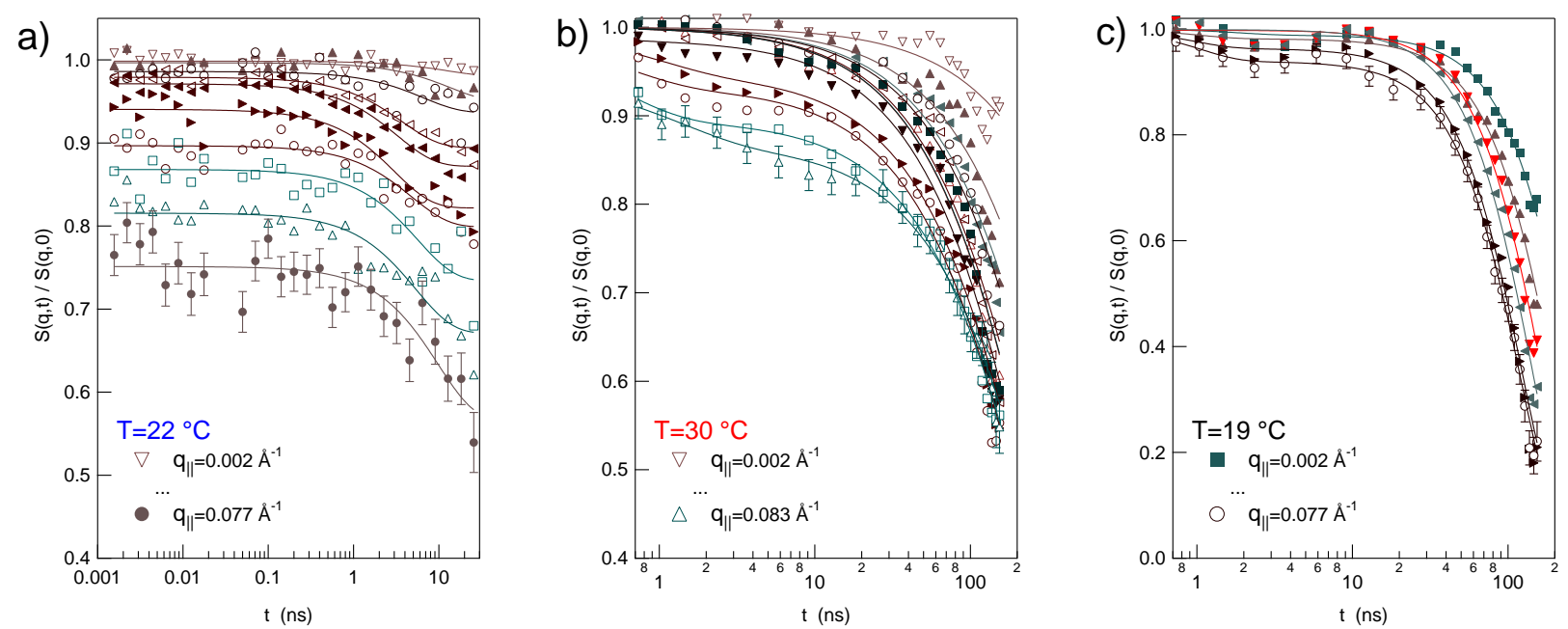

FIG. 2: (Color online). Intermediate scattering function $S(q, t) / S(q, 0)$ for selected $q \|$ values in the interval $0.002 \AA^{-1}<q_{\|}<0.8 \AA^{-1}$ measured on (a) IN11 at $\mathrm{T}=22^{\circ} \mathrm{C}$, just above the temperature of the main phase transition in deuterated DMPC -d54, (b) on IN15 at $30^{\circ} \mathrm{C}$, in the fluid phase of the phospholipid bilayers, and (c) on IN15, at $19^{\circ} \mathrm{C}$ in the gel phase. Two relaxation processes, one at about $10 \mathrm{~ns}$ and a second slower process at about 100ns are clearly distinguished. Solid lines in the Figures represent least-square fits to two exponential decays.

in Grenoble, France. Wavelength bands centered at $\lambda=7.4 \AA$ and $\lambda=14 \AA$ with $\Delta \lambda / \lambda \simeq 0.15$ (FWHM), respectively, have been set by a velocity selector. Partially (acyl chain) deuterated DMPC-d54 (1,2-dimyristoyl-snglycero-3-phoshatidylcholine) was obtained from Avanti Polar Lipids. Highly oriented multi lamellar membrane stacks of several thousands of lipid bilayers were prepared by spreading lipid solution of typically $25 \mathrm{mg} / \mathrm{ml}$ lipid in trifluoroethylene/chloroform (1:1) on 2" silicon wafers, followed by subsequent drying in vacuum and hydration from $\mathrm{D}_{2} \mathrm{O}$ vapor [17], resulting in a structure of smectic A symmetry. Twenty such wafers separated by small air gaps were combined and aligned with respect to each other to create a "sandwich sample" consisting of several thousands of highly oriented lipid bilayers (total mosaicity about $0.5^{\circ}$ ), with a total mass of about $400 \mathrm{mg}$ of deuterated DMPC. During the experiment, the samples were kept in a closed temperature and humidity controlled Aluminum chamber, and were hydrated from the vapor phase.

The layer fluctuations of lowest energy are the undulation modes, i.e., highly correlated (conformal) layer displacements which keep the inter-layer distances approximately constant. This conformality explains the strong modulation of the diffuse scattering in $q_{z}$, i.e., the Bragg sheet structure 11], corresponding to the diffuse tails (at $q_{\|}>0$ ) of the specular Bragg peaks $\left(q_{z}=n 2 \pi / d, q_{\|}=0\right)$. We have now measured spinecho curves on the first Bragg sheet as a function of $q_{\|}$, i.e., at constant $q_{z}=2 \pi / d$. A sketch of the scattering geometry is shown in Fig. 1(a). The in plane component of the scattering vector is calculated to $\mathrm{q}_{\|}=\mathrm{q}_{z} \tan (\omega)$, with $\mathrm{q}_{z} \approx 0.11 \AA^{-1}$ for the first reflectivity Bragg peak. $\omega$ is the rocking angle, i.e., the sample rotation with respect to specular Bragg angle. Figure 1(b) depicts a rocking curve in the fluid phase at $\mathrm{T}=30^{\circ} \mathrm{C}$ on the first reflectivity Bragg peak where the sharp Bragg component and the diffuse Bragg sheet can be well distinguished. In the 
inset, (c), a reflectivity curve is plotted. Peaks are distinctly broadened by the wavelength distribution.

Figure 2 shows the intermediate scattering function $\mathrm{S}\left(\mathrm{q}_{\|}, \mathrm{t}\right)$ for selected $\mathrm{q}_{\|}$values for spin-echo times of $0.001 \mathrm{~ns}<\mathrm{t}<20 \mathrm{~ns}$ for IN11 and 0.01ns $<\mathrm{t}<200 \mathrm{~ns}$ for IN15. Data have been taken at three different temperatures, at $19^{\circ} \mathrm{C}$, in the gel (ripple, $\mathrm{P}_{\beta^{\prime}}$ ) phase of the phospholipid bilayers, at $22^{\circ} \mathrm{C}$, just above the temperature of the main transition in deuterated DMPC-d54 (at $\mathrm{T}_{m} \approx 21.5^{\circ} \mathrm{C}$ ), and at $30^{\circ} \mathrm{C}$, far in the fluid $\mathrm{L}_{\alpha}$ phase of the membranes and above the regime of so-called anomalous swelling. The corresponding lamellar $d$ spacings were $d=56 \AA, 60 \AA$ and $54 \AA$ (gel, $22^{\circ} \mathrm{C}$ and fluid), respectively. Two relaxation processes, one at about 10ns and a second, slower process at about 100ns are clearly distinguished. Solid lines in the Figure represent least-square fits of the data to two stretched exponential decays, $S\left(q_{||}, t\right) / S\left(q_{||}, 0\right)=\left(A_{1}-A_{2}\right) \exp \left[-\left(t / \tau_{1}\left(q_{||}\right)\right)^{\beta 1}\right]+y_{2}+$ $\left(A_{2}-y_{2}\right) \exp \left[-\left(t / \tau_{2}\left(q_{\|}\right)\right)^{\beta 2}\right]$. The fitting results for $\beta_{1,2}$ were in the range of $0.96<\beta_{1,2}<1$ for the temperatures $\mathrm{T}=22^{\circ} \mathrm{C}$ and $30^{\circ} \mathrm{C}$, corresponding essentially to single exponential relaxations. Contrarily, the gel phase results $\left(19^{\circ} \mathrm{C}\right)$ give values of $\beta_{1,2} \approx 1.75$, i.e., compressed exponentials. Structural inhomogeneities and heterogeneous interactions would lead to a local relaxation dynamics and to stretched $(\beta<1)$ exponentials. A compressed exponential decay is incompatible with a diffusive, fluid like motion of the particles and might therefore be an intrinsic property of the gel state. The relaxation rates $\tau_{1}^{-1}$ and $\tau_{2}^{-1}$ in the gel and the fluid phase are depicted in Fig. 3 (a) and (b), after compilation of all measured $\mathrm{q}_{\|}$values on the two spectrometers. Also shown is the fast relaxation process for $\mathrm{T}=22^{\circ} \mathrm{C}$. Both relaxation branches are clearly dispersive. The fast process shows a $\mathrm{q}_{\| \mid}^{2}$ increase at small $\mathrm{q}_{\|}$values and a bend at about $\mathrm{q}_{\|} \approx 0.015 \AA^{-1}$. The dispersion in the gel phase and close to the phase transition in Fig. 3 (b) appear to be more pronounced as compared to $30^{\circ} \mathrm{C}$ dispersion. As a remarkable feature, a soft mode appears in the $\mathrm{T}=22^{\circ} \mathrm{C}$ dispersion, indicating a significant softening of the bilayer at a well defined wave number. The slow branches at $\mathrm{T}=19^{\circ} \mathrm{C}$ and $30^{\circ} \mathrm{C}$ also show increasing relaxation rates with increasing $\mathrm{q}_{\|}$ values, but with a distinct non-polynomial behavior.

The dispersion relation of the fluid $\mathrm{L}_{\alpha}$ phase lends itself to a quantitative comparison with theory. As an effect of the stacking of the bilayers and the substrate, undulation modes in lamellar phases decay with relaxation rates $\tau^{-1}\left(q_{\|}\right)=\kappa /\left(\eta_{3} d\right) q_{\|}^{2}$, while fluctuations of a free, non supported, bilayer would decay with $\tau^{-1} \propto q_{\|}^{3}[3,16]$. Generally, undulation modes are probed at $\mathrm{q}_{z}$ values of $q_{z}=2 \pi / d$. If the scattering is probed at finite components $\delta q_{z}:=\left(q_{z}-2 \pi / d\right)$ or measured with a relaxed $\mathrm{q}_{z}$ resolution, baroclinic modes rather than pure undulations are probed. Dynamic light scattering experiments can be described according to bulk smectic elasticity the-

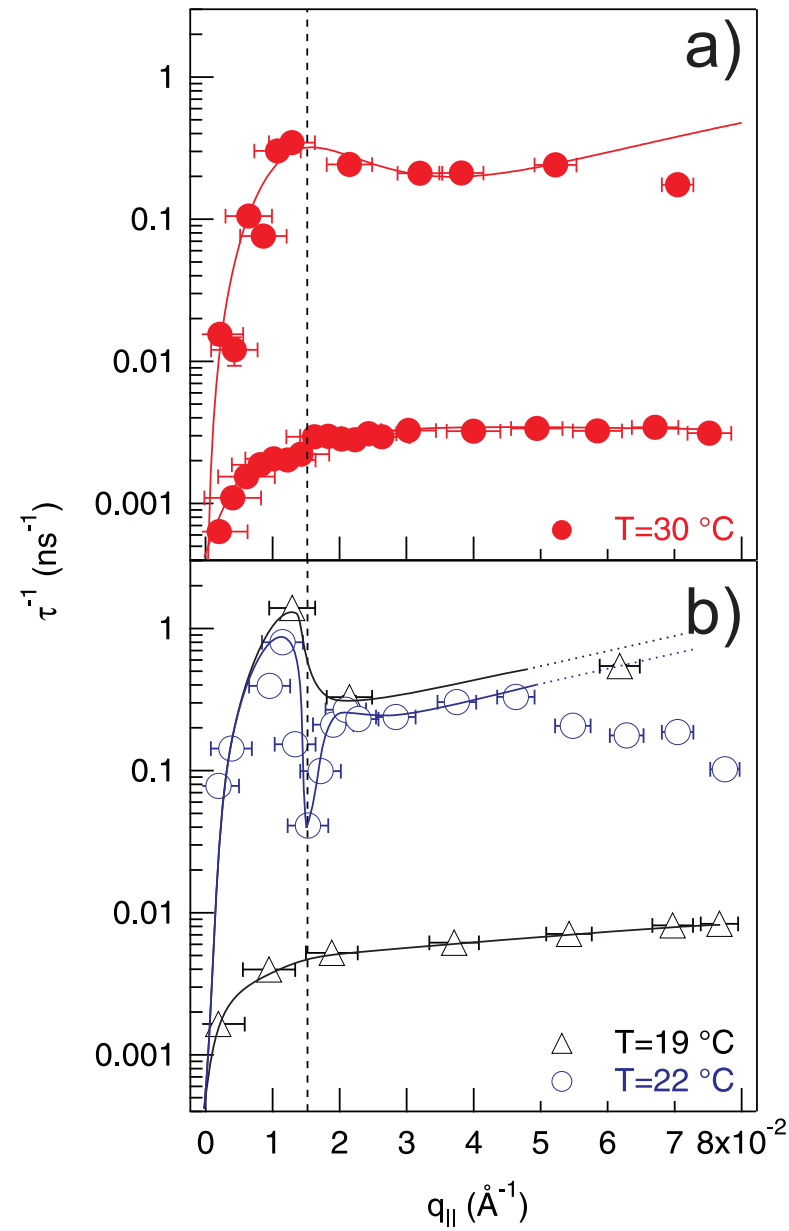

FIG. 3: (Color online). (a) Dispersion relations at $\mathrm{T}=30^{\circ} \mathrm{C}$. The solid line is a fit to Eq. (31). (b) Dispersion relations in the gel $\left(19^{\circ} \mathrm{C}\right)$ and in the fluid phase $\left(22^{\circ} \mathrm{C}\right)$. A pronounced soft mode is observed at $\mathrm{q}_{0} \approx 0.015 \AA^{-1}$ at $22^{\circ} \mathrm{C}$ (dotted vertical line). Solid lines in (b) are guides to the eye.

ory and multi lamellar fluctuations as a function of both symmetry axis $\left(q_{||}, q_{z}\right)$ by the dispersion relation [18]: $\tau^{-1}\left(q_{\|}\right)=q_{\|}^{2} \frac{B q_{z}^{2}+(\kappa / d) q_{\|}^{4}}{\eta_{3} q^{4}+\frac{1}{\mu} q_{z}^{2}}$. The transport coefficient $\mu$ was estimated to $\mu=d^{2} /\left(12 \eta_{0}\right)$ with $\eta_{0}$ the viscosity of the the solvent (water) [19]. Note that higher order elastic terms have been neglected. The neutron scattering experiments are carried out in the first Brillouin zone so that we have to replace $\mathrm{q}_{z}$ by $\delta q_{z}$, the deviation from the first zone center. However, finite size or resolution effects lead to a broadening of the diffuse Bragg sheet and therefore set a lower cut-off in the reachable $\delta q_{z}$. Following the idea of Ribotta 19] the above equation is then replaced by

$$
\tau^{-1}\left(q_{\|}\right)=\frac{\kappa / d}{\eta_{3}} q_{\| \mid}^{2} \frac{q_{\|}^{4}+(\pi /(\Lambda D))^{2}}{q_{\|}^{4}+\frac{1}{\mu \eta_{3}}(\pi / D)^{2}} .
$$

In our case, the $\mathrm{q}_{z}$ width of the diffuse scattering is not defined the finite size $D$ of the lipid film, but comes from 
the rather broad instrumental resolution, which is given by the wavelength spread. From the measured width $\Delta q_{z}$ of the first reflectivity Bragg peak in Fig. 11 (c) of $\Delta q_{z} \simeq 0.015 \AA^{-1}$ (HWHM, Lorentzian fit), we obtain an effective finite-size cutoff-length $D=\pi / \Delta q_{z} \simeq 212 \AA$ that we used to fit the measured fluid dispersion relation to Eq. (3), see solid line in Fig. 3 (a). The $\mathrm{q}_{z}$ component of the scattering vector was neglected, $q=\sqrt{q_{z}^{2}+q_{\|}^{2}} \approx q_{\|}$, because it may average out for symmetric broadening. Alternatively, the results can be compared to the theory given in 2] for thin film samples, including the boundary effects and surface tension. However, this theory gives dispersion relations for each of the $(N-1)$ eigenmodes, which makes a comparison difficult if not impossible without proper weighting of the modes.

Using Eq. (3), the following results are obtained for the three free parameters: $\kappa=14.8 \pm 8 \mathrm{k}_{B} \mathrm{~T}, \Lambda=$ $10.3 \pm 2.3 \AA, \eta_{3}=0.016 \pm 0.0006 \mathrm{~Pa}$ s. $\mathrm{B}$ is calculated to $\mathrm{B}=1.0810^{7} \mathrm{~J} / \mathrm{m}^{3}(d=54 \AA)$. Note that the value for $\Lambda$ compares quite well with the value obtained by a very different approach but for a similar swelling state [20]. Experiments at different osmotic pressures point to a distinct effect of the swelling state to the compressional modulus $B$ and $\Lambda$, consequently [7]. The bending modulus compares quite well to results from Molecular Dynamics [5] although the simulations do not reach the small $\mathrm{q}_{\|}$ values that we probe experimentally. $\kappa$ has large errors, but lies in the middle of the even larger range of literature values [22]. $\mathrm{q}_{\|}$values of $\mathrm{q}_{\|}>0.05 \AA^{-1}$ in Fig. 3 (a) and (b) show declining relaxation rates which deviate from the theoretic curve in Fig. 3 (a). The diffuse Bragg sheet was slightly bend for the high $\mathrm{q}_{||}$values and the corresponding diffuse scattering was likely dominated by defect and no longer purely thermal diffuse scattering with consequences for the precision in the determination of $\kappa$ and $\eta_{3}$. The dynamics at low $q_{||}$values is governed by the interplay of viscosity and inertia, i.e., $\tau^{-1} \propto \eta_{3} / \rho q_{\|}^{2}$, while at higher $\mathrm{q}_{\|}$values, the undulation dynamics becomes predominant. The difficulty in quantifying relaxation times at higher $\mathrm{q}_{\|}$values thus limits the precision in the determination of $\kappa$ distinctly. This constraint can be overcome in future studies with optimized set-ups and sample preparation. Note that conceptually, fluctuations in the gel phase, should not be described by the dispersion relations of fluid smectic A phases. The solid lines in Fig. 3 (b) are therefore guides to the eye.

In summary, NSE provides unique access to collective dynamics of shape fluctuations in solid supported multilamellar lipid membranes. The dispersion relation of the fast branch with relaxation rates between 1 and 10ns can be attributed mainly to undulation dynamics with the expected mixing of baroclinic modes resulting from a correction for finite resolution. The resulting effective sliding viscosity of the membrane system $\eta_{3}$ was found to be 16 times higher than that of water. The slow disper- sion branch with relaxation rates of about 100ns may be attributed to a surface relaxation mode 21] and will be addressed in a forthcoming publication. Furthermore a quite localized deviation from the undulation branch was observed at $\mathrm{T}=22^{\circ} \mathrm{C}$ just above the main phase transition, indicating the softening of a well defined wave number of $\mathrm{q}_{0} \approx 0.015 \AA^{-1}$. From this result, we speculate that the well known softening of phospholipid membrane upon approaching the main phase transition temperature from the fluid phase, i.e., the regime of "critical swelling" or "anomalous swelling" [22], occurs on a well defined length scale, here at $2 \pi / q_{0} \approx 420 \AA$. We have additionally measured the elastic scattering in the ripple phase and from the satellite peaks of the diffuse Bragg sheet we determine a ripple periodicity of $d_{r} \approx 130 \AA$, distinctly smaller than the length scale of the soft mode observed here. However, $d_{r}$ grows significantly at the transition, as we have measured by atomic force microscopy. Therefore, we speculate that the soft mode in the fluid $L_{\alpha}$ phase is linked to the formation of the ripple structure in the $P_{\beta^{\prime}}$ phase, but this remains to be investigated in more detail.

Acknowledgements: It is a pleasure to thank B. Farago and P. Fouquet for assistance at IN15 and IN11, and the ILL for allocation of ample beam time. T.S. acknowledges helpful discussions with D. Constantin. M.C.R enjoyed discussions with E. Kats and D. Bicout.

* Electronic address: rheinstaedter@ill.fr

[1] U. Seifert and S. Langer, Europhys. Lett. 23, 71 (1993).

[2] V. Romanov and S. Ul'yanov, Phys. Rev. E 66, 061701 (2002).

[3] A. Zilman and R. Granek, Phys. Rev. Lett. 77, 4788 (1996).

[4] R. Lipowsky and E. Sackmann, eds., Structure and Dynamics of Membranes, vol. 1 of Handbook of Biological Physics (Elsevier, North-Holland, Amsterdam, 1995).

[5] E. Lindahl and O. Edholm, Biophys. J. 79, 426 (2000).

[6] C. R. Safinya et al., Phys. Rev. Lett. 57, 2718 (1986).

[7] H. I. Petrache et al., Phys. Rev. E 57, 7014 (1998).

[8] A. Caillé, C.R. Acad. Sci. B 274, 891 (1972).

[9] N. Lei, C. Safinya, and R. Bruinsma, J. Phys. II 5, 1155 (1995);Ph.D. Dissertation, Rutgers (1993).

[10] Y. Lyatskaya et al., Phys. Rev. E 63, 011907 (2001).

[11] T. Salditt, M. Vogel, and W. Fenzl, Phys. Rev. Lett. 90, 178101 (2003).

[12] M. C. Rheinstädter et al., Phys. Rev. Lett. 93, 108107 (2004).

[13] M. F. Hildenbrand and T. M. Bayerl, Biophys. J. 88, 3360 (2005).

[14] I. Sikharulidze et al., Phys. Rev. Lett. 91, 165504 (2003); Phys. Rev. E 72, 011704 (2005).

[15] W. Pfeiffer et al., Europhys. Lett. 8, 201 (1989);23, 457 (1993).

[16] T. Takeda et al., J. Phys. Chem. Solids 60, 1375 (1999).

[17] C. Münster et al., Europhys. Lett. 46, 486 (1999).

[18] E. Freyssingeas, D. Roux, and F. Nallet, J. Phys. II France 7, 913 (1997). 
[19] R. Ribotta, D. Salin, and G. Durand, Phys. Rev. Lett. 32, 6 (1974).

[20] C. Ollinger et al., Europhys. Lett. 71, 311 (2005).

[21] H. Bary-Soroker, H. Diamantand, Langmuir 19, 1716
(2003).

[22] G. Pabst et al., Langmuir 19, 1716 (2003). 